\title{
ANALISIS COMPARATIVO DEL CURRÍCULO DE LA ASIGNATURA DE ORGANIZACIÓN ESCOLAR EN DIFERENTES UNIVERSIDADES DE LA COMUNIDAD DE MADRID
}

\author{
Tamara MUÑOZ NIETO ${ }^{1}$ \\ Eladio SEBASTIAN HEREDERO ${ }^{2}$
}

RESUMEN: En esta investigación se recoge un análisis sobre la forma de organizar la asignatura de organización del centro escolar en diferentes universidades de la Comunidad de Madrid. La asignatura de organización del centro escolar es una asignatura de carácter troncal y se imparte en los estudios de magisterio. Para un alumno universitario, en el caso de las universidades públicas, su centro de estudio depende de una nota. Por lo tanto se puede preguntar cómo hubiera sido su formación en otra universidad. Con esta investigación se trata de comparar los objetivos, metodología y forma de evaluar así como los materiales utilizados.

PALABRAS CLAVE: Organización escolar. Currículo. Programación.

\section{Estado de la Cuestión}

La organización escolar abarca muchos campos así pueda verse esta conceptualización elaborada: "Noción técnica y administrativa que trata de reagrupar y articular los diversos elementos que concurren en el funcionamiento interno de un Sistema Escolar (construcciones, programas escolares, personal docente, material didáctico, exámenes, investigación pedagógica, etc.)”.

Haciendo un breve recorrido histórico Pedro de Alcántara $^{3}$ en 1900 entendía por Organización escolar “[...] el conjunto de las disposiciones oficiales de carácter general y de medios particulares de acción por cuya virtud se establecen y aplican los principios, las leyes y las practicas porque se rigen las escuelas en general y particularmente consideradas [...]" años después en 1912 Blanco $^{4}$ decía que “[...] la Organización de las escuelas es la buena disposición de los elementos necesarios para educar o instruir a los niños [...]”; y más adelante García $\mathrm{Hoz}^{5}$ en 1964 “[...] la Organización Escolar será la ordenación de los distintos elementos de la escuela hecha con el fin de que todos concurran adecuadamente a la educación de los escolares.”

\footnotetext{
${ }^{1}$ Universidad de Alcalá . Alumna de $3^{\circ}$ de Magisterio. Madrid - Alcalá de Henares - España - 28801. tmuoz61@gmail.com

${ }^{2}$ Universidad de Alcalá. Departamento de Didáctica. Madrid - Alcalá de Henares - España. 28801 eladio.sebastian@uah.es

${ }^{3}$ Tomado de Lorenzo Delgado (1997).

${ }^{4}$ Tomado de Lorenzo Delgado (1997).

${ }^{5}$ Tomado de Martin Bris (1996).
} 
Pero entre las más técnicas nos quedamos con la definición de Gairín y Darder (2001, p.54) “ [...] El estudio de la intervención más adecuada de los elementos que intervienen en una realidad escolar con vistas a conseguir la realización de un proyecto educativo [...]” y la aportación de Manuel Lorenzo Delgado (1997, p.23) que es altamente integradora. “Teorías de la escuela desde la perspectiva de la ordenación de sus elementos en orden a un fin educativo/instructivo.”

En cualquier caso nos va a interesar conocer y analizar como se trabajan en esta disciplina esos elementos para el buen desarrollo posterior a su estudio universitario de la tarea docente.

\section{Objetivo de la investigación}

Esta investigación pretende ayudar a conocer cómo cambia la formación del futuro profesor de una universidad a otra. Un alumno podría haber accedido a cualquiera de estas universidades ya que se encuentran en la misma Comunidad y es interesante estudiar como hubiera cambiado la formación, ya no solo en esta asignatura sino en el resto.

Con esta investigación se ha querido profundizar en la asignatura de Organización del centro escolar (OCE), y comparar los programas de las diversas universidades de la Comunidad de Madrid. El objetivo es estudiar y comparar los programas de la asignatura para así sacar conclusiones de cómo se imparte esta asignatura en diferentes contextos. Analizaremos para ello distintos aspectos:

a) Carácter de la asignatura (tipo de asignatura: troncal, obligatoria...)

b) Curso en el que se imparte la asignatura. Es interesante investigar si esta asignatura requiere conocimientos previos, es decir si es recomendable impartirla en tercero de carrera o por el contrario los conocimientos previos no importan y se puede impartir en primero.

c) Temario y programación del temario. Investigar sobre cómo cada profesor o universidad organiza su temario. Y que preferencia se le da a cada tema.

d) Metodología. Como se trabaja la asignatura en general y lo más interesante como se trabaja cada tema, trabajo personal, trabajo en grupo, trabajo con lecturas....

e) Asistencia, valorar como de importante es la asistencia en esta asignatura y como se valora la misma. 
f) Evaluación, valorar la manera de calificar esta parte va muy unida a la metodología ya que dependiendo de la misma se calificara de una manera u otra. Es importante fijarse en la importancia que se le da a cada uno de los trabajos propuestos.

g) Bibliografía, se marca un especial interés en los libros recomendados por cada profesor par la asignatura, se podrán observar los libros claves y fundamentales y también las preferencias de cada profesor.

El objetivo es analizar todos estos aspectos por separado para luego poder elaborar una conclusión extraída de los resultados de cada centro universitario.

\section{Instrumentos para conocer lo que quiero}

La principal fuente de información va a ser el análisis de los programas de esta asignatura en las diferentes universidades, que encontramos en las web las mismas, todos ellos referentes al curso 2008-2009 a través de una guía de registro. También vamos a necesitar ayuda de las páginas web de las universidades a estudiar, sobre todo de las privadas para conocer a que universidad pública están adscritas y qué relación tiene con ella, por tanto un análisis documental amplio.

Pero como no todos los programas son igual de completos también se ha necesitado la ayuda de unas entrevistas (de los propietarios de los programas) pero se ha tratado de una entrevista informal donde se han recogido más bien impresiones personales.

\section{La muestra}

El contexto en el nos vamos a mover va a ser algunas de las universidades públicas y privadas de la Comunidad Autónoma de Madrid más importantes. Por tanto, una muestra significativa tanto por la importancia y prestigio de las Universidades como por el número de alumnos matriculados en las mismas.

Hay que señalar que, tal y como aparecen en este estudio, en la actual configuración del sistema de educación universitario los estudios de Magisterio se corresponden con una Diplomatura, por tanto no son estudios de Grado. Existen además estudios de Licenciaturas que sí son de Grado.

\section{UNIVERSIDADES PÚBLICAS}

Universidad Complutense de Madrid 
Es una Universidad pública. Situada en el campus de Moncloa (ciudad universitaria) cerca del centro de la ciudad de Madrid. Turnos de mañana y tarde.

Imparte las siguientes especialidades en la Facultad de Educación: Maestro especialidad en: Educación Física, Educación Social, Educación Infantil, Audición y lenguaje, Lengua Extranjera; Educación Primaria, Educación Musical y licenciaturas en Psicopedagogía y Pedagogía.

Las dos licenciaturas imparten una asignatura llamada Organización y Gestión de Centros Educativos con grandes similitudes a nuestra asignatura de estudio a diferencia de que se trata de una asignatura anual de 9 créditos.

Programa a estudiar de OCE de Magisterio: Lengua Extranjera y Educación Física.

\section{Universidad Autónoma de Madrid}

Es una Universidad pública. Situada en el campus de Cantos Blancos, localidad de Tres Cantos. Cuenta con turnos de mañana y tarde en casi todas sus especialidades.

Imparte las siguientes especialidades en la Facultad de Educación: Maestro especialidad en: Educación Física, Educación Infantil, Audición y Lenguaje, Lengua Extranjera, Educación Primaria, Educación Musical y licenciaturas en Psicopedagogía y CC de la Actividad física y el deporte.

En la licenciatura de Psicopedagogía imparten una asignatura con cierta relación a la referente en nuestro estudio es Calidad y Evaluación del sistema educativo. En la de Actividad física y deporte no se hace mención a ninguna asignatura parecida o relacionada.

Programa a estudiar de OCE de Magisterio de Educación Primaria

\section{Universidad de Alcalá de Henares}

La Universidad de Alcalá es una Universidad pública que tiene tres campus: dos de ellos en Alcalá de Henares y otro en Guadalajara (Fuera de la Comunidad Autónoma de Madrid). Cuenta con turno de mañana y las optativas se imparten en el turno de tarde.

Imparte las siguientes especialidades; Maestro especialidad en: Educación Física, Educación Infantil, Lengua Extranjera, Educación Primaria, Educación Musical, la escuela de Magisterio se encuentra en el campus de Guadalajara, y la licenciatura en Psicopedagogía que se imparte en el campus de Alcalá. 
En la licenciatura nombrada se imparte una asignatura con cierta relación a la referente en nuestro estudio es Evaluación de centros y programas con 6 créditos.

Programa a estudiar de OCE de Magisterio común con todas las especialidades.

\section{UNIVERSIDADES PRIVADAS}

\section{Escuela Universitaria de Magisterio “Escuni”}

Es una Escuela de Magisterio privada, sin ánimo de lucro, adscrita a la Universidad Complutense de Madrid. Fue creada en 1970 al unirse, en una sola institución, diversas Escuelas Normales de la Iglesia Católica. Actualmente, once instituciones de reconocido prestigio en el mundo de la educación y el Arzobispado de Madrid unen sus esfuerzos en este proyecto común.

Situada en Carabanchel, barrio del sur de Madrid, cuenta con horario de mañana y tarde para casi todas las especialidades.

Imparte las siguientes especialidades, Maestro especialidad en: Educación Física, Educación infantil, Lengua extranjera, Educación primaria, Educación musical y Educación especial.

Programa a estudiar de OCE de Magisterio común con todas las especialidades.

\section{El Centro Superior de Estudios Universitarios “La Salle”}

Inició su andadura en la localidad de Griñón (Madrid) en 1948 como centro de formación del profesorado. En 1970 traslada su sede a su emplazamiento actual en Madrid, y desde 1978 se encuentra oficialmente adscrito a la Universidad Autónoma de Madrid, por la que otorga títulos universitarios homologados y oficiales.

Imparte las siguientes especialidades, Maestro especialidad en: Educación Física, Educación Infantil, Lengua Extranjera, Educación Primaria, Educación Musical, Audición y Lenguaje, Educación Social y como licenciaturas cuentan con la de Psicopedagogía.

Programa a estudiar de OCE de Magisterio común con todas las especialidades.

\section{Analisis comparativos de resultados}


Una vez descritas las universidades a estudiar comenzamos a analizar los resultados obtenidos:

\section{Características generales de la asignatura}

Aspectos a tratar: curso en el que se imparte, número de créditos, carácter de la asignatura, duración y necesaria asistencia.

Tabla 1 - Características generales de la asignatura

\begin{tabular}{|l|l|l|l|l|l|}
\hline & Complutense & Autónoma & UAH & Escuni & La Salle \\
\hline $\begin{array}{l}\text { Curso en el } \\
\text { que se } \\
\text { imparte }\end{array}$ & $3^{\circ}$ & $3^{\circ}$ & $3^{\circ}$ & $3^{\circ}$ & $3^{\circ}$ \\
\hline $\begin{array}{l}\mathrm{N}^{\mathrm{o}} \text { de } \\
\text { créditos }\end{array}$ & 4,5 & 4,5 & 4,5 & 4,5 & 5 \\
\hline $\begin{array}{l}\text { Carácter de } \\
\text { la } \\
\text { asignatura }\end{array}$ & Troncal & Troncal & Troncal & Troncal & Troncal \\
\hline Duración & Cuatrimestral & Cuatrimestral & Cuatrimestral & Anual & Cuatrimestral \\
\hline Asistencia & $\mathrm{Si}$ & $\mathrm{Si}$ & $\mathrm{Si}$ & $\mathrm{Si}$ & --------- \\
\hline
\end{tabular}

Objetivos de la asignatura

En este apartado los tres programas coinciden y oscilan entre 6 y 9 objetivos que se pueden resumir en los siguientes (con alguna matización).

- $\quad$ Conocer y valorar las características y cometidos de los elementos básicos de la Organización Escolar.

- Relacionar y disponer adecuadamente los diversos elementos que intervienen en la organización de los Centros, así como las estructuras organizativas y de dirección que posibiliten el eficaz desarrollo del currículo y la práctica docente.

- Familiarizar al alumno con el análisis y elaboración de los instrumentos organizativos que configuran la dinámica de un Centro.

- Diseñar la aplicación de los diversos elementos y estructuras de la organización escolar a situaciones organizativas específicas.

- $\quad$ Conocer y ejercitar diversas técnicas que favorezcan el trabajo en equipo, la participación, la delegación y asunción de responsabilidades, la toma de decisiones, la resolución de conflictos y problemas.

- $\quad$ Conocer la evaluación y mejora de la calidad de un Centro Escolar. 


\section{Los contenidos temáticos}

Cada universidad los divide a su manera a continuación voy a presentar los bloques temáticos de las universidades a estudiar que oscilan entre 8 y 12.

Tabla 2 - Características generales de la asignatura

\begin{tabular}{|c|c|c|c|c|c|}
\hline $\begin{array}{l}\text { BLOQUE } \\
\text { TEMÁTICO }\end{array}$ & Complutense & Autónoma & UAH & Escuni & La Salle \\
\hline $\begin{array}{l}1 \\
\text { Introducción a } \\
\text { la materia. }\end{array}$ & $\begin{array}{l}\text { 1Principales } \\
\text { paradigmas de } \\
\text { la Organización } \\
\text { educativa } \\
\text { escolar }\end{array}$ & $\begin{array}{l}\text { 1Organización } \\
\text { escolar } \\
\text { 3Las } \\
\text { instituciones } \\
\text { educativas } \\
\text { como } \\
\text { organización }\end{array}$ & $\begin{array}{l}\text { 1La escuela } \\
\text { como } \\
\text { organización: } \\
\text { naturaleza } \\
\text { dimensiones y } \\
\text { enfoques } \\
\text { teóricos para } \\
\text { su } \\
\text { comprensión. }\end{array}$ & $\begin{array}{l}\text { 1Introducció } \\
\text { n a la } \\
\text { Organizació } \\
\text { n escolar }\end{array}$ & $\begin{array}{l}\text { 1La } \\
\text { organización } \\
\text { del centro } \\
\text { escolar }\end{array}$ \\
\hline $\begin{array}{l}2 \\
\text { Sistema } \\
\text { Educativo }\end{array}$ & $\begin{array}{l}\text { 3Política } \\
\text { educativa } \\
\text { escuela }\end{array}$ & $\begin{array}{l}\text { 2El sistema } \\
\text { educativo } \\
\text { español }\end{array}$ & $\begin{array}{l}\text { 3. La } \\
\text { estructura: } \\
\text { unidades } \\
\text { organizativas } \\
\text { básicas de las } \\
\text { escuelas y } \\
\text { estructura } \\
\text { organizativa }\end{array}$ & $\begin{array}{l}\text { 2Estructuras } \\
\text { organizativa } \\
\mathrm{s}\end{array}$ & $\begin{array}{l}\text { 2La población } \\
\text { escolar }\end{array}$ \\
\hline $\begin{array}{l}3 \\
\text { Planificación }\end{array}$ & $\begin{array}{l}\text { 6El proyecto } \\
\text { educativo de } \\
\text { centro. }\end{array}$ & $\begin{array}{l}\text { 4Organización } \\
\text { y planificación } \\
\text { general de las } \\
\text { instituciones } \\
\text { educativas. } \\
\text { 5Organización } \\
\text { de los alumnos } \\
\text { 6Organización } \\
\text { del } \\
\text { profesorado } \\
\text { 7Organización } \\
\text { del espacio } \\
\text { escolar } \\
\text { 8Organización } \\
\text { del tiempo } \\
\text { escolar }\end{array}$ & $\begin{array}{l}\text { 4Procesos de } \\
\text { planificación } \\
\text { institucional: } \\
\text { objetivos y } \\
\text { planes. }\end{array}$ & $\begin{array}{l}\text { 3Documento } \\
\text { s que } \\
\text { definen al } \\
\text { centro } \\
\text { escolar } \\
\text { 4Organizaci } \\
\text { ón de los } \\
\text { recursos } \\
\text { humanos. } \\
\text { 5Organizaci } \\
\text { ón de los } \\
\text { recursos } \\
\text { materiales. } \\
\text { 6Organizaci } \\
\text { ón del } \\
\text { tiempo. }\end{array}$ & $\begin{array}{l}\text { 5La } \\
\text { tecnología }\end{array}$ \\
\hline $\begin{array}{l}4 \\
\text { Calidad }\end{array}$ & $\begin{array}{l}\text { 4Organización } \\
\text { de las relaciones }\end{array}$ & $\begin{array}{l}\text { 12La } \\
\text { investigación } \\
\text { en la } \\
\text { organización } \\
\text { educativa. }\end{array}$ & $\begin{array}{l}\text { 5Los procesos } \\
\text { de mejora: la } \\
\text { cultura y el } \\
\text { clima. El } \\
\text { asesoramiento } \\
\text { y la } \\
\text { formación. }\end{array}$ & & $\begin{array}{l}\text { 6La calidad } \\
\text { de los centros } \\
\text { educativos } \\
\text { 3El ambiente }\end{array}$ \\
\hline $\begin{array}{l}5 \\
\text { Participación }\end{array}$ & $\begin{array}{l}\text { 7La } \\
\text { participación } \\
\text { ene los centros } \\
\text { educativos. }\end{array}$ & $\begin{array}{l}\text { 9La } \\
\text { participación y } \\
\text { el sistema de } \\
\text { relaciones en }\end{array}$ & $\begin{array}{l}\text { 2El contexto. } \\
\text { Agentes que } \\
\text { forman parte } \\
\text { del entorno. El }\end{array}$ & & $\begin{array}{l}4 \text { Las } \\
\text { relaciones }\end{array}$ \\
\hline
\end{tabular}




\begin{tabular}{|c|c|c|c|c|c|}
\hline & & $\begin{array}{l}\text { las } \\
\text { instituciones } \\
\text { educativas. }\end{array}$ & $\begin{array}{l}\text { sistema } \\
\text { educativo. } \\
\text { comunidad } \\
\text { educativa y } \\
\text { participación }\end{array}$ & & \\
\hline $\begin{array}{l}6 \\
\text { Evaluación }\end{array}$ & $\begin{array}{l}\text { 8La evaluación } \\
\text { de los centros } \\
\text { escolares. }\end{array}$ & $\begin{array}{l}\text { 10La } \\
\text { evaluación en } \\
\text { las } \\
\text { instituciones } \\
\text { educativas }\end{array}$ & $\begin{array}{l}\text { 6Los procesos } \\
\text { de dirección y } \\
\text { evaluación en } \\
\text { la } \\
\text { organización } \\
\text { escolar. }\end{array}$ & $\begin{array}{l}\text { 8Evaluación } \\
\text { de centros. }\end{array}$ & \\
\hline $\begin{array}{l}7 \\
\text { Otros }\end{array}$ & $\begin{array}{l}\text { 2Los centros } \\
\text { educativos y los } \\
\text { conflictos. } \\
\text { Modelos de } \\
\text { autoridad. } \\
\text { 5El conflicto en } \\
\text { la organización. }\end{array}$ & $\begin{array}{l}\text { 11Servicios } \\
\text { complementari } \\
\text { os }\end{array}$ & $\begin{array}{l}\text { 7La dimensión } \\
\text { de procesos de } \\
\text { dirección y } \\
\text { evaluación en } \\
\text { la } \\
\text { organización } \\
\text { escolar. }\end{array}$ & $\begin{array}{l}\text { 7Organizaci } \\
\text { ón de } \\
\text { actividades } \\
\text { especiales }\end{array}$ & $\begin{array}{l}\text { 7Marketing } \\
\text { educativo }\end{array}$ \\
\hline
\end{tabular}

\section{Metodología y evaluación}

Estas dos partes de la asignatura pueden ir juntas ya que la evaluación se realiza en torno a la metodología.

Tabla 3 - Metodología y evaluación

\begin{tabular}{|c|c|c|}
\hline & METODOLOGÍA & EVALUACIÓN \\
\hline Complutense & $\begin{array}{l}\text { Los temas se explicaran en clase } \\
\text { por medio de diapositivas y se } \\
\text { trabajaran de forma personal } \\
\text { mediante textos. }\end{array}$ & $\begin{array}{l}\text {-Entrega de trabajo compuesto } \\
\text { de trabajos individuales, parejas } \\
\text { y grupo. } \\
\text {-resumen crítico de un libro a } \\
\text { elegir. }\end{array}$ \\
\hline Autónoma & $\begin{array}{l}\text { Los temas se desarrollaran de } \\
\text { diferentes maneras: exposición } \\
\text { de los mismos en clase a manos } \\
\text { del profesor, exposición de } \\
\text { algunos de los temas a mano de } \\
\text { los alumnos (introducción de las } \\
\text { TIC) }\end{array}$ & $\begin{array}{l}\text {-examen escrito } \\
\text {-intervenciones orales en clase. } \\
\text {-utilización de las TIC } \\
\text {-proyectos y trabajos escritos } \\
\text {-defensa y presentación del } \\
\text { trabajo en el aula. }\end{array}$ \\
\hline \multirow[t]{2}{*}{ UAH } & $\begin{array}{l}\text { Los temas se explicaran en clase } \\
\text { por medio de diapositivas y } \\
\text { material práctico como distintos } \\
\text { documentos de centros. }\end{array}$ & $\begin{array}{l}\text { Existen dos formas de evaluar. } \\
\text { Ambas tiene en común la } \\
\text { realización de un portafolio: } \\
\text { - Examen de los contenidos } \\
\text { explicados en clase. } \\
\text {-Realizar un trabajo de } \\
\text { investigación. }\end{array}$ \\
\hline & $\begin{array}{l}\text { Clases intercaladas entre las } \\
\text { explicaciones del profesor y el } \\
\text { trabajo personal de los alumnos } \\
\text { mediante grupos. } \\
\text { Clases basadas en el diálogo. }\end{array}$ & $\begin{array}{l}\text {-evaluación continua: exámenes, } \\
\text { lecturas propuestas, trabajo en } \\
\text { equipo (escrito y oral), y } \\
\text { participación en clase. }\end{array}$ \\
\hline La Salle & $\begin{array}{l}\text { Mayoría de las clases expuestas } \\
\text { por el profesor. Intervención de } \\
\text { los alumnos con sus propuestas } \\
\text { de trabajo. }\end{array}$ & $\begin{array}{l}\text { Examen, trabajo individual y } \\
\text { trabajo en grupo. }\end{array}$ \\
\hline
\end{tabular}




\section{Bibliografía}

Este último apartado es para fijarnos en la importancia que le dan los profesores a ciertos libros y cuales son lecturas recomendadas por todas las universidades. Se ha de destacar los más significativos.

Tabla 4 - Bibliografía

\begin{tabular}{|c|c|c|c|c|c|}
\hline TÍTULO & Complutense & Autónoma & UAH & Escuni & $\begin{array}{l}\text { La } \\
\text { Salle }\end{array}$ \\
\hline $\begin{array}{l}\text { La organización escolar. } \\
\text { Práctica y fundamentos de } \\
\text { Antúnez y Gairín. }\end{array}$ & 1 & 1 & 1 & 1 & \\
\hline $\begin{array}{l}\text { Organización de centros } \\
\text { educativos de Gairín y Darder. }\end{array}$ & 1 & & 1 & 1 & \\
\hline $\begin{array}{l}\text { Organización y planificación } \\
\text { integral de centros. Ed infantil, } \\
\text { primaria y secundaria de Martín } \\
\text { Bris. }\end{array}$ & 1 & & & 1 & 1 \\
\hline $\begin{array}{l}\text { La organización y gestión del } \\
\text { centro educativo. Análisis de } \\
\text { casos prácticos de Lorenzo. }\end{array}$ & & 1 & 1 & & 1 \\
\hline $\begin{array}{l}\text { TOTAL de bibliografía } \\
\text { propuesta. }\end{array}$ & 34 & 12 & 35 & 40 & 33 \\
\hline
\end{tabular}

\section{Conclusiones}

Podemos observar a partir de los contenidos de la tabla 2 que esta es una asignatura muy práctica y real. Es decir que se trabajan los contenidos fundamentales teóricos de la asignatura según nos señalan las definiciones vistas y que se relacionan con lo que ocurre en los centros educativos cada día. Pero también influye en el grado de aceptación de esta asignatura que a primera vista parece interesante y útil, depende de la manera de impartirla, los bloques de contenidos tienen una cierta variabilidad, aunque hay un bloque común bastante amplio. Aquí hemos podido comprobar, también, como con unos mismos objetivos, la metodología y forma de evaluación puede variar el grado de participación e interés de los alumnos.

Con la tabla 1 podemos ver como en las cinco universidades escogen el último curso de la carrera para impartir esta asignatura, no ocurre lo mismo con otras asignaturas (por 
ejemplo las de especialidad de inglés no se cursan en el mismo curso como pueden ser Morfosintaxis, lengua inglesa, y didáctica de la legua inglesa).

Esta asignatura requiere un conocimiento previo de la normativa, del funcionamiento del centro e incluso un primer contacto con el propio centro antes de ser impartida. Estos conocimientos se van adquiriendo en las diversas asignaturas durante los dos primeros años y ya en el tercero sobre esa base se construye la “Organización del centro escolar”.

Con referencia al carácter de la misma vemos que tiene mucho peso en el currículum siendo la mayoría de 4,5 créditos y de carácter troncal, lo que da idea de la importancia que tiene en el conjunto de la formación.

El curso en el que se imparte la asignatura es en tercero ya que esta asignatura requiere un conocimiento previo de la normativa y del funcionamiento del centro.

En definitiva los objetivos son más o menos los mismos, pero la verdadera diferencia viene a la hora de conseguir esos objetivos.

El temario, metodología, evaluación y asistencia depende de la universidad pero en su gran mayoría dan mucha importancia la realización de trabajos por parte de los alumnos. Y la asistencia es importante para aquellas universidades en que la evaluación no sólo se basa en un examen final.

Existe una gran similitud tanto en los temas como en la forma de organizarlos de las tres primeras universidades: Complutense, Autónoma, Alcalá y Escuni. La Salle es la única que nombra de una forma muy diferente a sus temas aunque viendo la subdivisión de los mismos coincide con el resto. La evaluación educativa es uno de los últimos temas sino el último y todos comienzan con el concepto de organización escolar.

En esta parte es donde más se notan las diferencias entre las universidades y realmente es de lo que los alumnos más se percatan. En las tres universidades públicas no realizan examen, evalúan a través de los trabajos realizados en clase contando la asistencia como dato importante, con excepción de la UAH que da al posibilidad de realizar un examen o una trabajo de investigación, en los dos casos se le da mucha importancia y es requisito para pasar al asignatura realizar el portfolio. La universidad de La Salle es la que más importancia le da al examen y la que menos a la asistencia.

Hay que destacar que dependiendo del programa lo realizado en la clase es igual o no, queremos decir que si el programa está realizado por el departamento en general incluyendo a todos los profesores que imparten la materia en las distintas especialidades, varia más de la realidad a si el programa es individualizado de un solo profesor que imparte su asignatura en una o varias especialidades. 
Existe en bibliografía un conjunto de libros, que llamaremos básicos, que se trabajan en todas las universidades, aunque no todas proponen el mimo número de libros, existe una gran diferencia entre unas y otras.

En esta tabla podemos comprobar que ningún libro es elegido por las cinco universidades, es decir no hay un libro maestro que guste a todos los profesores, pero si coinciden principalmente en estos títulos.

También podemos destacar como hay universidades que sobrecargan de información a los alumnos proponiendo 40 libros de consulta y en cambio otras solo señalan una lista de apenas 12 para centrar más el tema.

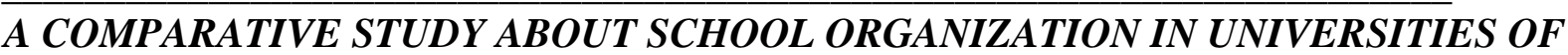
MADRID, SPAIN

ABSTRACT: In this investigation I analyze the way to teach the subject "Organización del centro escolar" in different Comunidad de Madrid"s universities. With this investigation we can compare the objectives, the methodology and the way to evaluate and even materials that they use. The "Organizacion del centro escolar"'s subject is main and it is teaching in the education schools. For a university student, in the case of public universities; his study center depends of a calcification. So he can ask himself: how could be his formation in another university.

KEYWORDS: School organization. Curriculum. Planning.

\section{REFERENCIAS}

ANTUNEZ, S. Y.; GAIRÍN, J. La organización escolar: práctica y fundamentos. Barcelona: Grao, 2000.

ENCICLOPEDIA virtual de didáctica y organización escolar. Disponible en: <http://www.pangea.org/peremarques/evdioe.htm>. Acceso en: 05 mayo 2009.

GAIRIN, J.; DARDER, P. Organización de centros educativos. Barcelona: CISSPRAXIS, 2001.

MARTIN BRIS, M. Organización y planificación integral de centros: educación infantil, primaria y secundaria. Madrid: Escuela Española, 1996.

LORENZO DELGADO, M. La organización y gestión del centro educativo: análisis de casos prácticos. Madrid: Universitas, 1997.

LOVELACE, M. Gestión y organización del centro escolar ante los cambios sociales y 
culturales: nuevas funciones, nuevas estructuras organizativas. In: SEMINARIO LA FORMACIÓN DEL PROFESORADO EN EDUCACIÓN INTERCULTURAL, 2002, Barcelona. Anales... Disponible en:

<http://www.aulaintercultural.org/article.php3?id_article=268>. Acceso en: 05 mayo 2009.

UNIVERSIDAD AUTÓNOMA DE MADRID. Disponible en: <http://www.uam.es/>. Acceso en: 05 mayo 2009.

UNIVERSIDAD COMPLUTENSE DE MADRID. Disponible en: <http://www.ucm.es/> . Acceso en: 06 jun. 2009.

UNIVERSIDAD DE ALCALÁ DE HENARES. Disponible en: < http:www.uah.es>. Acceso en: 07 jul. 2009.

UNIVERSIDAD ESCUNI DE MADRID. Disponible en: < http://www.ucm.es/escuni>. Acceso en: 06 jun. 2009.

UNIVERSIDAD LA SALLE DE MADRID. Disponible en:

$<$ http://www.eulasalle.com/Inicio.aspx>. Acceso en: 06 jun. 2009. 